PROCEEDINGS OF THE

AMERICAN MATHEMATICAL SOCIETY

Volume 128, Number 11, Pages 3379-3390

S 0002-9939(00)05453-8

Article electronically published on May 18, 2000

\title{
AN $n$-DIMENSIONAL SPACE THAT ADMITS \\ A POINCARÉ INEQUALITY BUT HAS NO MANIFOLD POINTS
}

\author{
BRUCE HANSON AND JUHA HEINONEN
}

(Communicated by Albert Baernstein II)

\begin{abstract}
For each integer $n \geq 2$ we construct a compact, geodesic metric space $X$ which has topological dimension $n$, is Ahlfors $n$-regular, satisfies the Poincaré inequality, possesses $\mathbb{R}^{n}$ as a unique tangent cone at $\mathcal{H}_{n}$ almost every point, but has no manifold points.
\end{abstract}

\section{INTRODUCTION}

In this paper, we shall construct, for each integer $n \geq 2$, a metric space $X$ with the following properties:

(i) $X$ is compact, geodesic, and of topological dimension $n$;

(ii) $X$ has Hausdorff dimension $n$ and the Hausdorff $n$-measure $\mathcal{H}_{n}$ satisfies the Ahlfors regularity condition:

$$
\frac{1}{C} R^{n} \leq \mathcal{H}_{n}\left(B_{R}\right) \leq C R^{n}
$$

for all balls $B_{R}$ in $X$ of radius $R<\operatorname{diam~} X$ and for some $C \geq 1$ independent of the ball;

(iii) $X$ admits the Poincaré inequality: there is a constant $C \geq 1$ such that

$$
\int_{B}|u-m v(u, B)| d \mathcal{H}_{n} \leq C \operatorname{diam} B \int_{B} \rho d \mathcal{H}_{n}
$$

for all integrable continuous functions $u$ in a ball $B$ and for all upper gradients $\rho$ of $u$ in $B$, where $m v(u, B)$ denotes the mean-value of $u$ in $B$;

(iv) $X$ possesses $\mathbb{R}^{n}$ as a unique tangent cone at $\mathcal{H}_{n}$ almost every point;

(v) $X$ has no manifold points.

An additional property is that $X$ is uniformly rectifiable in the sense of David and Semmes [DS]. A metric space is called geodesic if every pair of points in the space can be joined by a curve whose length is the distance between the points. A point in a metric space is a manifold point if it has a neighborhood that is homeomorphic

Received by the editors August 14, 1998 and, in revised form, January 18, 1999.

1991 Mathematics Subject Classification. Primary 43A85; Secondary 28A75.

Key words and phrases. Poincaré inequality, Ahlfors $n$-regular, manifold point.

The second author was supported by NSF grant DMS 96-22844.

(C)2000 American Mathematical Society 
to an open subset in Euclidean space. A tangent cone of a metric space $(X, d)$ at a point $p \in X$ is a pointed Gromov-Hausdorff limit of a sequence $\left(X, \varepsilon_{i}^{-1} d, p\right)$ as $\varepsilon_{i} \rightarrow 0$; cf. [GLP 3.E]. An upper gradient of a function $u$ in a metric space is any Borel function $\rho$, with values in $[0, \infty]$, such that

$$
|u(x)-u(y)| \leq \int_{\gamma} \rho d s
$$

for all pairs of points $x, y$ in the space and for all rectifiable curves $\gamma$ joining them. Observe that $\rho=|\nabla u|$ is an upper gradient of a smooth function $u$ on a Riemannian manifold.

Upper gradients and Poincaré inequalities in abstract metric spaces were introduced in $[\mathrm{HeK}]$ in connection with quasiconformal mappings. Recently, Cheeger $\mathrm{C}$ has exploited these concepts in studying differentiability of Lipschitz functions on metric spaces. (See $\mathrm{HaK} 2$ for further applications and references.) It was demonstrated in these papers that a lot of classical analysis, or first order calculus, can be accomplished in spaces that admit a Poincaré inequality, and a natural question therefore is: how pathological can a space be if (1.2) holds there?

Bourdon and Pajot $\mathrm{BP}]$ showed that there exist metrics on the Sierpinski sponge such that (1.2) holds and that the Ahlfors regularity condition (1.1) holds for some nonintegral dimension. Shortly afterwards, Laakso [L] exhibited spaces that are Ahlfors regular of any given dimension greater than one, and where (1.2) holds. The spaces constructed by Bourdon and Pajot, and by Laakso, are all topologically 1dimensional, and they have no manifold points. (Having heard of our construction, Laakso pointed out to us that his methods in $[\mathrm{L}]$ can be used to construct a space with properties (i) - (v). However, Laakso's spaces cannot be realized as compact subsets of some Euclidean space, up to a bi-Lipschitz change in the metric. It seems likely that our example has this property, but we have not checked the details.) The spaces that we construct in this paper have complicated local topology. One wonders if it is possible to have a space with properties (i) - (v) that is, say, locally contractible.

We thank Jeff Cheeger whose inquiries led us to think about the example presented in this paper.

\section{General Set-UP AND the main PROposition}

All measures in this paper are locally finite Borel measures, nontrivial on each open set.

Our construction of a metric space $X$ as in the Introduction is based on a proposition whose set-up is as follows:

$$
\text { for each nonnegative integer } m, X_{m}=\left(X_{m}, d_{m}, \mu_{m}\right)
$$

is a connected, compact metric measure space;

$$
X_{0} \subset X_{1} \subset X_{2} \subset \cdots \subset X_{\infty}=\bigcup_{m=0}^{\infty} X_{m},
$$

where the inclusion $X_{m} \hookrightarrow X_{m+1}$ is an isometric embedding; 


$$
\begin{gathered}
X_{m} \text { 's are uniformly doubling, that is, there exists } \\
\text { a constant } C \geq 1 \text {, independent of } m \text {, such that } \\
\mu_{m}\left(B_{m}(x, 2 r)\right) \leq C \mu_{m}\left(B_{m}(x, r)\right) \text { whenever } x \in X_{m} \text { and } r>0, \\
\text { where } B_{m}(x, r)=\left\{y \in X_{m}: d_{m}(x, y)<r\right\} \\
\qquad \mu_{m+1} \mid X_{m}=\mu_{m}
\end{gathered}
$$

there is a real-valued function $\varphi$, independent of $m$, such that

$$
\mu_{m}\left(B_{m}(x, r)\right) \leq \varphi(r) \text { for all } x \in X_{m} \text { and } r>0 .
$$

We can equip $X=X_{\infty}$ with a distance $d=d_{\infty}$, where

$$
d \mid X_{m}=d_{m}
$$

and with a Borel measure $\mu=\mu_{\infty}$, where

$$
\mu \mid X_{m}=\mu_{m} .
$$

It follows from (2.3) that the limiting space $(X, d, \mu)$ is doubling as well:

$$
\mu(B(x, 2 r)) \leq C \mu(B(x, r)),
$$

where $C \geq 1$ is as in (2.3) and $B(x, r)$ is a ball in $X$.

If the center of a ball is irrelevant, we use the notation $B(r)$.

Next we describe two additional assumptions for this general set-up. For each $m=0,1,2, \ldots$ let $A_{m}=X_{m} \backslash X_{m-1}$, where $X_{-1}=\emptyset$.

Assumption A. There exists a sequence $D_{0}, D_{1}, \ldots$ of measurable subsets of $X$ with the following properties: Given any $M>0$ we have

$$
\mu(B(y, r)) \leq C(M) \mu\left(B^{j}(y, r)\right)
$$

for all $y \in D_{j}$ and $r<M R_{j}(y)$, where $R_{j}(y)$ is the diameter of the $y$-component of $D_{j} \cup D_{j+1}$ and $B^{j}(y, r)$ is the $y$-component of $B(y, r) \cap\left(D_{j} \cup D_{j+1}\right)$. Given $a \in A_{\ell}, y \in A_{m}, m>\ell$, with $a$ and $y$ in the same component of $X_{m} \backslash X_{\ell-1}$, there are points $a=y_{\ell}, y_{\ell+1}, \ldots, y_{m}=y$ with $y_{j} \in D_{j}$ and positive numbers $r_{\ell}, r_{\ell+1}, \ldots, r_{m-1}$ such that

$$
r_{j}+r_{j+1}<C R_{j}=C R_{j}\left(y_{j}\right) \text { for } j=\ell, \ldots, m-2
$$

and $y_{j}$ and $y_{j+1}$ lie in the same component of $D_{j} \cup D_{j+1}$ for $j=\ell, \ldots, m-1$. Moreover,

$$
\sum_{j=\ell}^{m-1} r_{j} \leq C d(a, y)
$$

and

$$
d\left(y_{j}, y\right) \leq C r_{j}, \quad j=\ell, \ldots, m-1 .
$$

Above, $C \geq 1$ is a fixed constant (independent of $a, y$ ). 
Assumption B. Given $x \in A_{k}, y \in A_{m}$, there exists $\ell \leq \min \{k, m\}$ and $a \in A_{\ell}$ such that

$$
a \text { and } x \text { (resp. } a \text { and } y \text { ) lie in the same component of }
$$

$$
X_{k} \backslash X_{\ell-1} \text { (resp. } X_{m} \backslash X_{\ell-1} \text { ), }
$$

and that

$$
d(x, a)+d(a, y) \leq C d(x, y),
$$

where $C \geq 1$ is a fixed constant (independent of $x, y$ ). (Note that $a$ may equal $x$ or y.)

Before stating the main proposition, we define, for $R>0$, the truncated maximal function $M_{R} g$ of a (nonnegative) Borel function $g$ in $X$ by

$$
M_{R} g(x)=\sup _{r<R} m v(g, B(x, r)),
$$

where the notation $m v(g, E)$ denotes the mean value of $g$ over a measurable set $E$. In the proof of the proposition, we shall need maximal functions on subsets of $X$ that are components of $D_{j} \cup D_{j+1}$; these will be denoted by $M_{R}^{j}$, the component being understood from the context.

Proposition 2.15. Let Assumptions $\triangle$ and $B$ be satisfied. Suppose that $u$ and $\rho$ are measurable functions in $B(R)$, with $\rho$ nonnegative, satisfying

$$
|u(x)-u(y)| \leq C_{1} d(x, y)\left(M_{C_{2} d(x, y)}^{j} \rho(x)+M_{C_{2} d(x, y)}^{j} \rho(y)\right)
$$

for all $x, y \in B\left(R / C_{3}\right)$ that lie in the same component of $D_{j} \cup D_{j+1}$ for some $j$. Then

$$
|u(x)-u(y)| \leq C_{4} d(x, y)\left(M_{C_{5} R} \rho(x)+M_{C_{5} R} \rho(y)\right)
$$

for all $x, y \in B\left(R / C_{6}\right)$, where the constants $C_{i}$ for $4 \leq i \leq 6$ depend only on the constants $C_{i}$ for $1 \leq i \leq 3$ and on the constants appearing in Assumptions $A$ and $B$.

Proof. Fix $x, y \in B\left(R / C_{6}\right)$, where the constant $C_{6}$ will be determined shortly. Assume that $x \in A_{k}$ and $y \in A_{m}$. By Assumption $\mathrm{B}$, we may choose $a \in A_{\ell}$ for some $\ell \leq \min \{k, m\}$ such that (2.13) and (2.14) are satisfied. It will be apparent from the proof that we may assume $\ell<\min \{k, m\}$. We use Assumption $\AA$ and find $y_{\ell}, \ldots, y_{m}$ and $r_{\ell}, \ldots, r_{m-1}$ such that (2.10)-(2.12) hold. Therefore, we can fix $C_{6}$ large enough so that

$$
B^{j}=B^{j}\left(y_{j}, r_{j}\right) \subset B\left(R / C_{3}\right), \quad j=\ell, \ldots, m-1 .
$$

For convenience, we also define $B^{m}=\{y\}$ and $r_{m}=0$. Let $s_{j}=\min \left\{R_{j}, d(x, y)\right\}$. We claim that, given $\kappa>0$, there are points $y_{j}^{\prime} \in B^{j}$ such that

$$
M_{\kappa s_{j}}^{j} \rho\left(y_{j}^{\prime}\right) \leq N M_{C R} \rho(y), \quad j=\ell, \ldots, m-1,
$$

for some positive constants $N$ and $C$, which depend only on the data and on $\kappa$. Below we will choose $\kappa$ depending only on the data so that the same will be true for $N$ and $C$. To prove the claim, fix $j$ and let $\kappa>0$. Then for $r_{j} / 2 \leq r<\kappa s_{j}$, we have by (2.9), (2.8) and 2.12) that

$$
m v\left(\rho, B^{j}(z, r)\right) \leq C m v(\rho, B(y, C r)) \leq C M_{C R} \rho(y)
$$


for every $z \in B^{j}$ and for some $C$ depending only on the data. Therefore, if (2.19) fails to hold for some large $N$, then for every $z \in B^{j}, d\left(y_{j}, z\right)<r_{j} / 2$, there exists $r(z)<r_{j} / 2$ such that

$$
m v\left(\rho, B^{j}(z, r(z))\right)>N M_{C R} \rho(y)
$$

Thus, by using standard covering arguments and the fact that the measure $\mu$ is doubling on $D_{j} \cup D_{j+1}$ by (2.9), we obtain that

$$
m v\left(\rho, B^{j}\right)>C N M_{C R} \rho(y) .
$$

On the other hand, by (2.20),

$$
m v\left(\rho, B^{j}\right) \leq C M_{C R} \rho(y),
$$

which contradicts (2.21) for $N$ large enough. Thus, (2.19) follows.

Next, note that from (2.10), (2.11) and (2.14) we get

$$
r_{j}+r_{j+1}<C s_{j}, \quad j=\ell, \ldots, m-2 .
$$

From this inequality and (2.12), it follows that we can (and do) choose $\kappa$ such that

$$
C_{2} d\left(w_{j}, w_{j+1}\right) \leq \kappa s_{j}, \quad j=\ell, \ldots, m-1
$$

for all $w_{j} \in B^{j}$ and $w_{j+1} \in B^{j+1}$. Next, note that for $j=\ell, \ldots, m-2$ we have

$$
d\left(y_{j}^{\prime}, y_{j+1}^{\prime}\right) \leq C\left(r_{j}+r_{j+1}\right)
$$

by (2.12). Moreover, if we define $y_{m}^{\prime}=y$, we have $d\left(y_{m-1}^{\prime}, y_{m}^{\prime}\right) \leq C r_{m-1}$ so that

$$
\sum_{j=\ell}^{m-1} d\left(y_{j}^{\prime}, y_{j+1}^{\prime}\right) \leq C d(a, y)
$$

by (2.11). It follows from Assumption $\AA$ that $y_{j}^{\prime}, y_{j+1}^{\prime}$ lie in the same component of $D_{j} \cup D_{j+1}$ for $j=\ell, \ldots, m-1$. Therefore, by (2.18), (2.19), (2.22), and assumption (2.16)

$$
\begin{aligned}
\left|u\left(y_{j}^{\prime}\right)-u\left(y_{j+1}^{\prime}\right)\right| & \leq C d\left(y_{j}^{\prime}, y_{j+1}^{\prime}\right)\left(M_{C_{2} d\left(y_{j}^{\prime}, y_{j+1}^{\prime}\right.}^{j} \rho\left(y_{j}^{\prime}\right)+M_{C_{2} d\left(y_{j}^{\prime}, y_{j+1}^{\prime}\right.}^{j} \rho\left(y_{j+1}^{\prime}\right)\right) \\
& \leq C d\left(y_{j}^{\prime}, y_{j+1}^{\prime}\right) M_{C R} \rho(y)
\end{aligned}
$$

for all $j=\ell, \ldots, m-1$. It hence follows from (2.23) that

$$
\left|u\left(y_{\ell}^{\prime}\right)-u(y)\right| \leq C d(a, y) M_{C R} \rho(y)
$$

Similarly, we also have

$$
\left|u\left(x_{\ell}^{\prime}\right)-u(x)\right| \leq C d(a, x) M_{C R} \rho(x)
$$

for some $x_{\ell}^{\prime} \in B^{\ell}$. Finally, because $y_{\ell}^{\prime}$ and $x_{\ell}^{\prime}$ lie in the same component of $D_{\ell}$ with $d\left(x_{\ell}^{\prime}, y_{\ell}^{\prime}\right) \leq C d(x, y)$ by (2.11) and (2.14), we have by assumption (2.16) as in the proof of (2.19) that

$$
\left|u\left(x_{\ell}^{\prime}\right)-u\left(y_{\ell}^{\prime}\right)\right| \leq C d(x, y)\left(M_{C R} \rho(x)+M_{C R} \rho(y)\right) .
$$

The proposition now follows by combining (2.14), and (2.25)-(2.27). 


\section{THE EXAMPLE}

We now verify the claim made in the Introduction by constructing a metric space with properties (i) - (v). Before beginning our construction, we record the following two results.

Theorem 3.1. A doubling metric measure space $X$ admits a weak Poincaré inequality if and only if there is a constant $C \geq 1$ such that

$$
|u(x)-u(y)| \leq C d(x, y)\left(M_{R} \rho(x)+M_{R} \rho(y)\right)
$$

whenever $u$ is a continuous function in $B(R), x, y \in B(R / C)$, and $\rho$ is an upper gradient of $u$ in $B$.

Theorem 3.3. Let $X,\left(Y_{i}\right)$ be a countable collection of metric measure spaces that are uniformly Ahlfors $n$-regular (condition (1.1)). Suppose $\left(F_{i}\right)$ is a disjoint collection of compact subsets of $X$ such that each $F_{i}$ has an isometric copy (also called $F_{i}$ ) inside $Y_{i}$. Suppose further that $n \geq s>n-1$ and that

$$
\mathcal{H}_{s}^{\infty}\left(F_{i} \cap B(R)\right) \geq C^{-1} R^{s}
$$

for all balls $B(R)$ in $X$ or in $Y_{i}$ centered at $F_{i}$, where $C \geq 1$ is independent of $i$ and $0<R<\min \left\{\operatorname{diam} X\right.$, diam $\left.Y_{i}\right\}$. Then, if $X$ and $Y_{i}$ admit a weak Poincaré inequality with constants independent of $i$, then the space $X \cup_{\left(F_{i}\right)} Y_{i}$ admits a weak Poincaré inequality as well. The statement is quantitative in the usual sense.

Recall that a space admits a weak Poincaré inequality if (1.2) holds with the ball $B$ on the left hand side replaced by $\lambda B$ for some fixed $0<\lambda \leq 1$; cf. HeK] Section 5. In (3.4), $\mathcal{H}_{s}^{\infty}$ denotes the Hausdorff $s$-content of a set.

The metric space $X \cup_{\left(F_{i}\right)} Y_{i}$ is obtained by glueing each $Y_{i}$ to $X$ along $F_{i}$; the metric is given by $d(a, b)=\inf _{p \in F_{i}}\left(d_{Y_{i}}(a, p)+d_{X}(p, b)\right)$, if $a \in Y_{i}$ and $b \in X$, and by $d(a, b)=\inf _{p \in F_{i}, q \in F_{j}}\left(d_{Y_{i}}(a, p)+d_{X}(p, q)+d_{Y_{j}}(q, b)\right)$, if $a \in Y_{i}, b \in Y_{j}$, and $i \neq j$.

The maximal function characterization of the Poincaré inequality, Theorem 3.1 was proved in [HeK, Lemma 5.15] (it was assumed there that $X$ be locally compact, but this assumption is redundant). Theorem 3.3 was proved in [HeK, Theorem 6.15] for the case of two spaces $X, Y$ (again, the local compactness assumption is redundant). The more general case described in Theorem 3.3 easily reduces to the special case by way of the maximal function characterization in Theorem 3.1 Note that by using Theorem 3.3 alone it is relatively easy to construct spaces with the Poincaré inequality and large nonmanifold pieces. To obtain a space with no manifold points at all, we require an iterative construction together with a repeated use of Theorem 3.1 .

We now begin the required construction in earnest. Fix an integer $n \geq 2$. Next, fix a compact totally disconnected set $E \subset[0,1]$ such that

$$
|E|_{1} \geq \frac{2}{3}
$$

and that

$$
\mathcal{H}_{1 / 2}^{\infty}(E \cap B(r)) \geq C^{-1} r^{1 / 2}
$$


for each ball (interval) $B(r)$ centered at $E$ with $0<r \leq 1$. We leave it to the reader to construct such a set $E$. Here and below, we let $|\cdot|_{j}$ denote Lebesgue $j$-measure in $\mathbb{R}^{j}$.

Now let $I^{j}=[0,1]^{j}$, a closed $j$-cube in $\mathbb{R}^{j}$, and $F=E \times I^{n-1}$. Then $F$ is a compact subset of $I^{n}$ with empty interior satisfying

$$
|F|_{n} \geq \frac{2}{3}
$$

and

$$
\mathcal{H}_{n-\frac{1}{2}}^{\infty}(F \cap B(r)) \geq C^{-1} r^{n-\frac{1}{2}}
$$

for each $n$-ball $B(r)$ centered at $F$ with radius $0<r \leq \sqrt{n}$. By shrinking $F$ slightly, we may assume, in addition, that

$$
\operatorname{dist}\left(F, \partial I^{n}\right)=\delta>0 .
$$

Here and below, a cube will refer to a closed dyadic sub- $n$-cube of $I^{n}$. Given a cube, $Q$, we define $F_{Q}=T_{Q}(F)$, where $T_{Q}: I^{n} \rightarrow Q$ is the canonical similarity map onto $Q$; that is, $T_{Q}(x)=\ell(Q) x+z_{Q}$, where $\ell(Q)$ is the side length of $Q$ and $z_{Q}$ is the vertex of $Q$ which is closest to the origin. Then $F_{Q}$ is a compact subset of $Q$ with empty interior satisfying

$$
\begin{gathered}
\left|F_{Q}\right|_{n} \geq \frac{2}{3}|Q|_{n}, \\
\mathcal{H}_{n-\frac{1}{2}}^{\infty}\left(F_{Q} \cap B(r)\right) \geq C^{-1} r^{n-\frac{1}{2}}
\end{gathered}
$$

for each $n$-ball $B(r)$ centered at $F_{Q}$ with radius $0<r<\operatorname{diam} Q$, and

$$
\operatorname{dist}\left(F_{Q}, \partial Q\right)=\delta \ell(Q) \text {. }
$$

Define $X_{0}=I^{n}, \mu_{0}=$ Lebesgue $n$-measure restricted to $X_{0}$, and $d_{0}=$ the standard Euclidean distance in $\mathbb{R}^{n}$. Let $W_{1}=\left\{Q_{m}\right\}$ be a Whitney decomposition of $\mathbb{R}^{n} \backslash F$ restricted to $X_{0} \backslash F$; thus the cubes in $W_{1}$ are dyadic subcubes of $I^{n}$. (Compare [S, p. 16].) Then for each $Q, W_{1}$ determines a Whitney decomposition $W_{Q}$ of $Q \backslash F_{Q} ;$ namely, $W_{Q}=\left\{T_{Q}\left(Q_{m}\right): Q_{m} \in W_{1}\right\}$. Define $W_{2}=\bigcup_{Q \in W_{1}} W_{Q}$ and more generally for each integer $k \geq 1, W_{k+1}=\bigcup_{Q \in W_{k}} W_{Q}$. Let $W=\bigcup_{k=1}^{\infty} W_{k}$, and set $X_{1}=X_{0} \cup_{\left(F_{Q}\right)} Q^{\prime}$, where $Q^{\prime}$ is an identical copy of $Q$, and $\left(F_{Q}\right)$ is indexed by $Q \in W$.

As explained above after Theorem [3.3, there is a natural extension of $d_{0}$ to a metric $d_{1}$, which is geodesic in $X_{1}$. Similarly, $\mu_{0}$ extends naturally to a measure $\mu_{1}$ :

$$
\mu_{1}(K)=\mu_{0}\left(K \cap X_{0}\right)+\sum_{Q \in W} \mu_{0}\left(K \cap\left(Q^{\prime} \backslash F_{Q}\right)\right)
$$

for each Borel set $K \subset X_{1}$.

Thus, $X_{1}$ is $X_{0}$ together with a countable number of "flaps" glued on. Each flap is a cube $Q^{\prime}$ which is attached to $X_{0}$ at the compact interiorless set $F_{Q}$, a scaled version of the initial set $F$. Now to get $X_{2}$ from $X_{1}$, we glue flaps onto each flap in $X_{1}$ in exactly the same way that we glued flaps onto $X_{0}$ to create $X_{1}$. In other words, we replace each flap $Q^{\prime}$ by an appropriate scaled copy of $X_{1}$. Metric $d_{1}$ and measure $\mu_{1}$ extend to $d_{2}$ and $\mu_{2}$ in the canonical way. By repeating this process inductively, we create a sequence of compact and geodesic metric measure spaces 
$X_{m}=\left(X_{m}, d_{m}, \mu_{m}\right)$. We let $X=\bigcup_{m=0}^{\infty} X_{m}$ as in Section 2, note that (2.1), (2.2), and (2.4) are satisfied. We define the metric $d$ and measure $\mu$ on $X$ by formulas (2.6) and (2.7).

We shall show that $X$ satisfies properties (i) - (v) in the Introduction except for compactness; in the end, a simple argument will show that the completion of $X$ will satisfy all requirements. Observe that the size of the flaps goes down rapidly so that $\operatorname{diam} X \leq 2 \sqrt{n}$.

Properties (iv) and (v) are quite clear from the construction, for almost every point in $X$ belongs to some $F_{Q}$ or its iterative counterpart.

The next order of business is to show that (2.3) and (2.5) hold, so that all of the properties (2.1) - 2.8) in the basic set-up in Section 2 are satisfied. To that end, we actually show that

$$
\frac{1}{C} R^{n} \leq \mu_{m}(B(R)) \leq C R^{n}
$$

for each ball $B(R) \subset X_{m}, 0<R<\operatorname{diam} X_{m}$, and for some $C \geq 1$ independent of $m$ and $R$; in other words, the spaces $X_{m}$ are uniformly Ahlfors $n$-regular. This implies both (2.3) and (2.5). We shall show, in effect, that (3.13) holds with $X$ in place of $X_{m}$ and $\mu$ in place of $\mu_{m}$, but a similar argument gives (3.13).

To prove (3.13), we set up some notation. First, recall that for $k=0,1,2, \ldots$, $A_{k}=X_{k} \backslash X_{k-1}$, where $X_{-1}=\emptyset$. Given $x \in A_{k}, y \in A_{\ell}$, with $\ell \geq k$, we say that $y$ is a descendant of $x$ if there is a path connecting $x$ to $y$ in $X_{\ell} \backslash X_{k-1}$. We let $D(x)$ denote the set of all descendants of $x \in X$, and $D_{m}(x)=D(x) \cap X_{m}$.

Throughout the rest of this paper, $C$ denotes a positive constant that only depends on some obvious data.

To verify the lower bound in (3.13), note that if $x \in X$, then $x \in A_{m}$ for some $m \geq 0$. If $m=0$, the lower bound is trivial. If $m \geq 1$, then there is a sequence of flaps $Q_{1}^{\prime}, \ldots, Q_{m}^{\prime}$ that "connect" $X_{0}=Q_{0}$ to $x \in Q_{m}^{\prime}$. Because the diameter of these consecutive flaps decreases at least geometrically, we have that $\operatorname{dist}\left(Q_{j}^{\prime}, x\right) \leq$ $C$ diam $Q_{j}^{\prime}$ for each $j=0, \ldots, m$. Thus, if some $Q_{j}^{\prime} \subset B(x, R) \backslash B(x, R / 2)$, then diam $Q_{j}^{\prime} \geq R / C$, which implies the required lower bound; if not, there must be a cube $Q_{j}^{\prime}$ that either connects $\partial B(x, R / 2)$ to $\partial B(x, 3 R / 4)$, or $\partial B(x, 3 R / 4)$ to $\partial B(x, R)$, in which case the lower bound again holds.

Next, we shall verify the upper bound in (3.13). To that end, it suffices to show that

$$
\mu(B(x, R) \cap D(x)) \leq C R^{n}
$$

if $x \in X$ and $0<R<\operatorname{diam} X$. (Indeed, if $j$ is the smallest integer such that $B(x, R) \cap X_{j} \neq \emptyset$, then for $y \in B(x, R) \cap X_{j}$ we have $B(x, R) \subset B(y, 2 R) \cap D(y)$.) In order to prove (3.14), note first that for any cube $Q$ we have $\bigcup_{P \in W_{Q}} P=Q \backslash F_{Q}$, and hence (3.10) implies that

$$
\mu\left(\bigcup_{P \in W_{Q}} P\right) \leq \frac{1}{3} \mu(Q) .
$$

From this it follows that

$$
\mu\left(\bigcup_{Q \in W_{m+1}} Q\right) \leq \frac{1}{3} \mu\left(\bigcup_{Q \in W_{m}} Q\right) \leq \frac{1}{3^{m+1}} \mu\left(X_{0}\right)=\frac{1}{3^{m+1}}
$$


for $m=1,2, \ldots$, and hence that $\mu\left(\bigcup_{Q \in W} Q\right) \leq \frac{1}{2}$. Therefore

$$
\mu\left(X_{1} \backslash X_{0}\right) \leq \frac{1}{3} \mu\left(\bigcup_{Q \in W} Q\right) \leq \frac{1}{6} .
$$

From these estimates and from the construction of $X_{2}$, we obtain that

$$
\mu\left(X_{2} \backslash X_{1}\right) \leq \frac{1}{6} \mu\left(\bigcup_{Q \in W} Q\right) \leq \frac{1}{12}
$$

and, in general, that $\mu\left(X_{m} \backslash X_{m-1}\right) \leq \frac{1}{3}\left(\frac{1}{2}\right)^{m}$ for $m=1,2, \ldots$. It follows that

$$
\mu(X) \leq \frac{4}{3}=\frac{4}{3} \mu\left(X_{0}\right)
$$

and by self-similarity of the construction that

$$
\mu\left(D\left(Q^{\prime}\right)\right) \leq \frac{4}{3} \mu\left(Q^{\prime}\right)=\frac{4}{3} \mu(Q)
$$

for each $Q^{\prime}$ which is a flap at some stage of the construction, where $D\left(Q^{\prime}\right)=$ $\bigcup_{x \in Q^{\prime}} D(x)$.

Finally, to prove (3.14), we may assume that $x \in X_{0}$, by self-similarity of the construction. Then $D(x)=X$, and we must show that

$$
\mu(B(x, R)) \leq C R^{n} .
$$

It is easy to see that there is at most one $Q \in W$, call it $Q_{0}$, such that both $F_{Q_{0}} \cap B(x, R) \neq \emptyset$ and $B(x, R) \cap X_{0} \subset Q_{0}$. Let

$$
W^{*}=\left\{Q \in W: Q \neq Q_{0}, \quad F_{Q} \cap B(x, R) \neq \emptyset\right\} .
$$

Note that $W^{*}$ is nonempty. In addition, it follows from (3.12), and from the fact that none of the $Q$ 's in $W^{*}$ contain $B(x, R) \cap X_{0}$, that

$$
Q \subset B(x, C R)
$$

for each $Q \in W^{*}$. Let $W^{* *}$ be the collection of all maximal $Q$ 's from $W^{*}$; that is, $Q \in W^{* *}$ if $Q \subset P$ and $P \in W^{*}$ imply $Q=P$. If we denote, for $Q \in W^{* *}$, $E(Q)=\left\{P \in W^{*}: P \subset Q\right\}$, we have that

$$
\bigcup_{Q \in W^{* *}} E(Q)=\bigcup_{Q \in W^{*}} Q
$$

and that $E(Q) \cap E(P)=\emptyset$ if $Q \neq P$. Now for $Q \in W^{* *}$ the ratio of

$$
\mu\left(\left(\bigcup_{P \in E(Q), P \neq Q} D\left(P^{\prime}\right) \backslash Q\right)\right)
$$

and $\mu(Q)$ is less than or equal to the ratio of $\mu\left(X \backslash X_{0}\right)$ and $\mu\left(X_{0}\right)$, which does not exceed $\frac{1}{3}$ by (3.15). Also, since $\mu\left(D\left(Q^{\prime}\right) \backslash Q\right) \leq \frac{2}{3} \mu(Q)$ by (3.10) and (3.16), we have that

$$
\mu\left(\left(\bigcup_{P \in E(Q)} D\left(P^{\prime}\right) \backslash Q\right)\right) \leq \mu(Q) .
$$


Next, abbreviate $B=B(x, R)$. We note that

$$
B=\left(B \cap X_{0}\right) \cup\left(B \cap\left(D\left(Q_{0}^{\prime}\right) \backslash Q_{0}\right)\right) \cup\left(B \cap\left(\bigcup_{Q \in W^{*}} D\left(Q^{\prime}\right) \backslash Q\right)\right) .
$$

But the last of the three sets in this union belongs to

$$
\bigcup_{Q \in W^{*}} D\left(Q^{\prime}\right) \backslash Q=\bigcup_{Q \in W^{* *}}\left(\left(\bigcup_{P \in E(Q)} D\left(P^{\prime}\right) \backslash Q\right)\right),
$$

and so by (3.18), (3.19), and the disjointness of $W^{* *}$ we have that

$$
\begin{aligned}
\mu(B & \left.\cap\left(\bigcup_{Q \in W^{*}} D\left(Q^{\prime}\right) \backslash Q\right)\right) \leq \sum_{Q \in W^{* *}} \mu(Q) \\
& \leq \mu\left(B(x, C R) \cap X_{0}\right) \leq C R^{n}
\end{aligned}
$$

On the other hand,

$$
\mu\left(B \cap\left(D\left(Q_{0}^{\prime}\right) \backslash Q_{0}\right)\right) \leq \mu\left(B \cap\left(\bigcup_{Q \in W^{*}} D\left(Q^{\prime}\right) \backslash Q\right)\right) .
$$

Because clearly $\mu\left(B \cap X_{0}\right) \leq C R^{n}$, (3.17) follows from these last two inequalities.

The proof of the upper bound in (3.13) is now complete. In particular, we have verified assumptions (2.1) - 2.8) in the basic set-up.

Next we verify Assumption A, as described in Section 2 Define $\mathcal{Q}_{0}=\left\{X_{0}\right\}$ and for $m=1,2, \ldots$ let $\mathcal{Q}_{m}$ be the collection of all flaps $Q^{\prime}$ added at the $m$ th stage to get $X_{m}$ from $X_{m-1}$. So for $m=1,2, \ldots$ we have

$$
A_{m}=X_{m} \backslash X_{m-1}=\bigcup_{Q^{\prime} \in \mathcal{Q}_{m}}\left(Q^{\prime} \backslash F_{Q}\right)
$$

We define $D_{0}=X_{0}$ and $D_{m}=\bigcup_{Q^{\prime} \in \mathcal{Q}_{m}} Q^{\prime}$ for $m=1,2, \ldots$. Note that (2.9) follows from 3.14) and the fact that $\mu\left(B^{j}(y, r)\right) \geq C r^{n}$ for all $r<R_{j}(y)$.

Now assume $a \in A_{\ell}, y \in A_{m}, m>\ell$, and that $a$ and $y$ lie in the same component of $X_{m} \backslash X_{\ell-1}$. Then there is a sequence of flaps $Q_{\ell}^{\prime}, Q_{\ell+1}^{\prime}, \ldots, Q_{m}^{\prime}$ such that $a \in$ $Q_{\ell}^{\prime}, y \in Q_{m}^{\prime} \backslash F_{Q_{m}}$, and that each $Q_{j}^{\prime}$ is a flap at the $j$ th stage of construction, so that $Q_{j}^{\prime} \subset D_{j}$ and $\bigcup_{j=\ell}^{m} Q_{j}^{\prime}$ is connected. (If $\ell=0$, we allow $X_{0}$ as a flap.) Let $\gamma$ be a geodesic joining $a$ to $y$. Choose $y_{\ell}=a, y_{m}=y$ and $y_{j} \in \gamma \cap Q_{j}^{\prime}$ for $j=\ell+1, \ldots, m-1$. For $j=\ell, \ell+1$ choose

$$
r_{j}=\min \left\{\ell\left(Q_{j}^{\prime}\right), d(a, y)\right\}
$$

and for $j=\ell+2, \ldots, m-1$ choose $r_{j}=\ell\left(Q_{j}^{\prime}\right)$. Note that 2.10 follows easily from $R_{j} \geq \ell\left(Q_{j}^{\prime}\right) \geq \ell\left(Q_{j+1}^{\prime}\right)$. Moreover, by (3.20), (2.11) follows if we can show

$$
\sum_{j=\ell+2}^{m-1} r_{j} \leq C d(a, y)
$$


in particular, if $\ell+2 \geq m$, there is nothing to prove. Otherwise, each path connecting $a$ and $y$ must intersect both $F_{Q_{\ell+2}}$ and $\partial Q_{\ell+2}$; hence, by (3.12), we have $r_{\ell+2} \leq C d(a, y)$. Estimate (3.21) now follows from the exponential decrease of the $r_{j}^{\prime} s$ for $j>\ell+1$. Finally, (2.12) follows because, for $j>\ell+1$, we have

$$
d\left(y_{j}, y\right) \leq \sum_{i=j}^{m} \operatorname{diam} Q_{i}^{\prime} \leq C \operatorname{diam} Q_{j}^{\prime} \leq C r_{j}
$$

Assumption $\mathrm{A}$ is thus verified.

Assumption $\mathrm{B}$ is readily verified as follows: Given $x \in A_{k}$ and $y \in A_{m}$, take a geodesic $\gamma$ joining them, and define $\ell$ to be the smallest integer such that $\gamma$ meets $A_{\ell}$. Pick $a$ to be any point in $A_{\ell} \cap \gamma$. Then (2.13) and (2.14) hold; in fact $d(x, a)+d(a, y)=d(x, y)$.

After this preparation, we are ready to show that $X$ admits a Poincaré inequality as in (1.2). Proposition 2.15) is used here.

We shall verify condition (3.2) in $X$; then Theorem 3.1 implies that $X$ admits a weak Poincaré inequality. Because $X$ is doubling and geodesic, the main result in HaK 1] then implies that $X$ admits a Poincaré inequality as in (1.2). Now (3.2) follows from (2.16) by way of Proposition 2.15] But by Theorem 3.3 each component of $D_{j} \cup D_{j+1}$ satisfies a weak Poincaré inequality (with constants independent of $j$ ), so we infer from the necessity part of Theorem 3.1 that (2.16) indeed holds.

We have thus demonstrated that $X$ satisfies properties (i) - (v) except compactness. To achieve this last property, we simply consider the completion $\bar{X}$ of $X$. It is easy to see from the construction that $\bar{X}$ is totally bounded, hence compact, and geodesic. Finally, we extend the measure $\mu$ to $\bar{X}$ by setting $\mu(\bar{X} \backslash X)=0$. Upon changing the notation, therefore, we have verified all the properties (i) - (v) in the Introduction.

\section{ACNOWLEDGEMENT}

The authors thank the referee for pointing out an oversight in an earlier version of Proposition [2.15.

\section{REFERENCES}

[BP] M. Bourdon and H. Pajot, "Poincaré inequalities and quasiconformal structures on the boundary of some hyperbolic buildings", Proc. Amer. Math. Soc. 127 (1999), 2315-2324. MR 99j:30024

[C] J. Cheeger, "Differentiability of Lipschitz functions on metric measure spaces", GAFA, Geom. Funct. Anal. 9 (1999), 428-517.

[DS] G. David and S. Semmes, "Fractured fractals and broken dreams; self-similar geometry through metric and measure", Oxford Lecture Series in Mathematics and its Applications 7, Clarendon Press, Oxford, 1997. MR 99h:28018

[GLP] M. Gromov, "Structures Métriques pour les Variétés Riemanniennes", (J. Lafontaine and P. Pansu, eds.), Cedic/Fernand Nathan, Paris, 1981. MR 85e:53051.

[HaK 1] P. Hajlasz and P. Koskela, "Sobolev meets Poincaré", C.R. Acad. Sci. Paris 320 (1995), 1211-1215. MR 96f:46062

[HaK 2] P. Hajlasz and P. Koskela, "Sobolev met Poincaré", Mem. Amer. Math. Soc. (to appear).

$[\mathrm{HeK}]$ J. Heinonen and P. Koskela, "Quasiconformal maps in metric spaces with controlled geometry", Acta Math. 181 (1998), 1-61. MR 99j:30025 
[L] T.J. Laakso, "Ahlfors $Q$-regular spaces with arbitrary $Q$ admitting weak Poincaré inequality", GAFA, Geom. Funct. Anal. (to appear).

[S] E.M. Stein, "Singular Integrals and Differentiability Properties of Functions", Princeton University Press, Princeton, New Jersey, 1970. MR 44:7280

Department of Mathematics, St. Olaf College, Northfield, Minnesota 55057

E-mail address: hansonb@stolaf.edu

Department of Mathematics, University of Michigan, Ann Arbor, Michigan 48109 